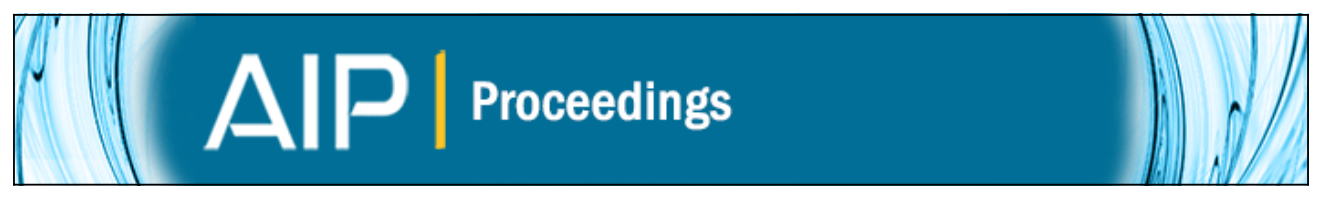

\title{
The First Stars: Formation of Binaries and Small Multiples
}

Athena Stacy, Thomas H. Greif, and Volker Bromm

Citation: AIP Conference Proceedings 1294, 289 (2010); doi: 10.1063/1.3518881

View online: http://dx.doi.org/10.1063/1.3518881

View Table of Contents:

http://scitation.aip.org/content/aip/proceeding/aipcp/1294?ver=pdfcov

Published by the AIP Publishing

Articles you may be interested in

WIMP Dark Matter and the First Stars

AIP Conf. Proc. 1294, 66 (2010); 10.1063/1.3518893

The Formation and Fragmentation of Primordial Protostellar Discs

AIP Conf. Proc. 1294, 52 (2010); 10.1063/1.3518890

The Impact of Ultraviolet Radiation on Secondary Pop III Star Formation

AIP Conf. Proc. 1294, 262 (2010); 10.1063/1.3518868

Three Modes of Metal-Enriched Star Formation in the Early Universe

AIP Conf. Proc. 1294, 110 (2010); 10.1063/1.3518837

FIRST Project: Formation and Feedback of the First Stars

AIP Conf. Proc. 990, 386 (2008); 10.1063/1.2905587 


\title{
The First Stars: Formation of Binaries and Small Multiples
}

\author{
Athena Stacy*, Thomas H. Greif ${ }^{\dagger}$ and Volker Bromm* \\ ${ }^{*}$ Department of Astronomy, University of Texas, Austin, TX 78712, USA \\ ${ }^{\dagger}$ Max-Planck-Institut für Astrophysik, Karl-Schwarzschild-Strasse 1, 85740 Garching bei \\ München, Germany
}

\begin{abstract}
We investigate the formation of metal-free, Population III (Pop III), stars within a minihalo at $z \simeq 20$, starting from cosmological initial conditions. We follow the collapsing gas in the center of the minihalo up to number densities of $10^{12} \mathrm{~cm}^{-3}$. We then study the protostellar accretion onto the initial hydrostatic core, which we represent as a growing sink particle. We continue our simulation for $5000 \mathrm{yr}$ after the first sink particle has formed. During this time, a disk-like configuration is assembled around the first protostar. At the end of the simulation, a small multiple system has formed within this disk, dominated by a binary with masses $\sim 40 \mathrm{M}_{\odot}$ and $\sim 10 \mathrm{M}_{\odot}$. If Pop III stars were to form typically in binaries or small multiples, the standard model of primordial star formation, where single, isolated stars are predicted to form in minihalos, would have to be modified.
\end{abstract}

We carry out our investigation using Gadget, a widely-tested three dimensional smoothed particle hydrodynamics (SPH) code [1,2]. Simulations are carried out in a periodic box with size of $100 \mathrm{~h}^{-1} \mathrm{kpc}$ (comoving) and initialized at $z=99$ with both dark matter particles and SPH gas particles, in accordance with a $\Lambda$ CDM cosmology. The evolution was followed up to gas densities of $10^{12} \mathrm{~cm}^{-3}$. We then represent these high-density peaks with sink particles. Sink particles are held constant at that density as they accrete surrounding gas particles.

$300 \mathrm{yr}$ after the first sink has formed, the gas further fragments, and a second sink is created. Concurrently, the central region evolves into a Keplerian disk. Disk instability later sets in, and spiral structure develops. Several new fragments then form through the Toomre instability and are represented by new sink particles. The disk spins up as the inner few hundred solar masses falls inward to smaller radii. The spirals and fragments serve to transport angular momentum outward through gravitational torques, allowing the inner gas to accrete onto the sinks. After $5000 \mathrm{yr}$, the disk has grown to a radial size of slightly less than 2000 AU (Fig. 1). The disk mass, which excludes the sinks, stays fairly constant at $\sim 35 \mathrm{M}_{\odot}$ as gas falling from the outer regions onto the disk replaces the material accreted onto the sinks at similar rates.

The main sink approximately grows as $\mathrm{M} \propto \mathrm{t}^{0.55}$ (Fig. 2a). By $5000 \mathrm{yr}$ after its formation, it has grown to $\sim 43 \mathrm{M}_{\odot}$. The initial accretion rates are similar to those expected from analytic solutions $[3,4,5]$ as well as the accretion rate found in [6]. The rate then declines approximately as $\dot{M} \propto \mathrm{t}^{-0.45}$. Extrapolating to later times, the protostar represented by the main sink can easily accrete several hundred solar masses over its main sequence lifespan of $3 \times 10^{6}$ yr. Future work will explore how protostellar feedback will affect these results. For more details, see [7]. 

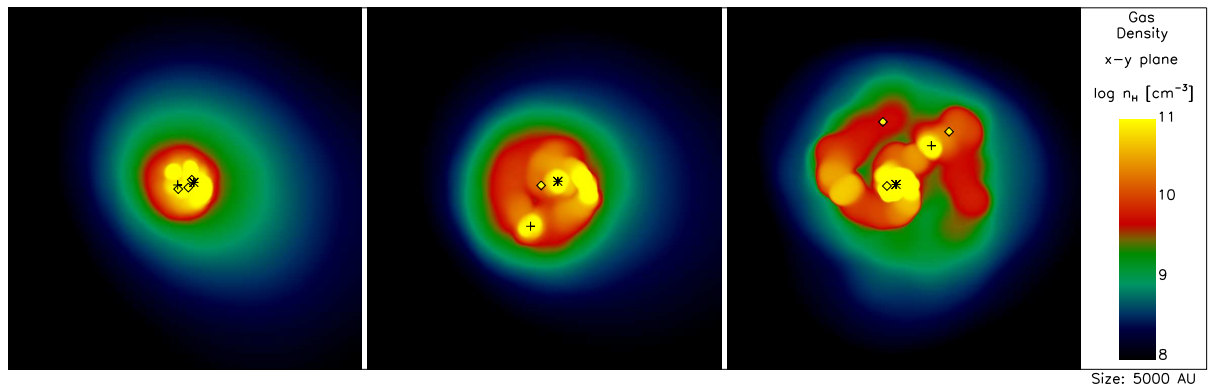

FIGURE 1. Density projection of the central high-density region (length $5000 \mathrm{AU}$ ), shown at $1000 \mathrm{yr}$ (left), $2000 \mathrm{yr}$ (center), and $5000 \mathrm{yr}$ (right) after the initial sink formation. Asterisk: location of the initial and most massive sink. Cross: location of second most massive sink. Diamonds: locations of the other sinks.
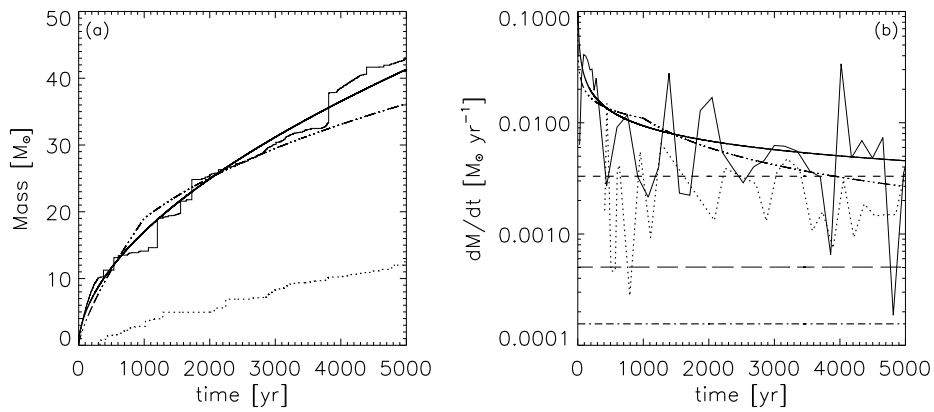

FIGURE 2. (a) Thin solid line: mass of the first sink particle over time in our calculation. Thick solid line: least-squares power-law fit to the sink mass over time. Dash-dot line: mass evolution obtained by [6]. Dotted line: mass growth of the second largest sink. (b) Thin solid line: accretion rate of the main sink particle throughout our calculation. Thick solid line: power-law fit to the mass accretion rate. Dashdouble dot line: instantaneous accretion rate found by [6]. Dotted line: accretion rate of the second largest sink. Double dash-single dot line: average accretion rate found by [6] over the lifetime of a Pop III star, determined by extrapolating their rate to $3 \times 10^{6} \mathrm{yr}$. Short-dash line and long-dash line: Shu accretion rates for isothermal gas at $700 \mathrm{~K}$ and $200 \mathrm{~K}$, respectively.

\section{REFERENCES}

1. V. Springel, N. Yoshida, and S. D. M. White, New Astron. 6, 79-117 (2001).

2. V. Springel, and L. Hernquist, MNRAS 333, 649-664 (2002).

3. R. B. Larson, MNRAS 145, 271-295 (1969).

4. M. W. Penston, MNRAS 144, 425-448 (1969).

5. F. H. Shu, ApJ 214, 488-497 (1977).

6. V. Bromm, and A. Loeb, New Astron. 9, 353-364 (2004).

7. A. Stacy, T. H. Greif, and V. Bromm, MNRAS 403, 45-60 (2010). 